\title{
Serum D-dimer levels at admission for prediction of outcomes in acute pancreatitis
}

\author{
Jianhua Wan ${ }^{\dagger}$, Xiaoyu Yang ${ }^{\dagger}$, Wenhua He, Yin Zhu, Yong Zhu, Hao Zeng, Pi Liu, Liang Xia ${ }^{*}$ (D) and Nonghua Lu
}

\begin{abstract}
Background: Systemic alterations in coagulation are associated with complications of acute pancreatitis (AP). D-dimer, a fibrin degradation product, was recently described as a marker of pancreatitis outcome. Early prediction is essential for reducing mortality in AP. The present study aims to assess the relationship between elevated serum D-dimer levels and the severity of AP.
\end{abstract}

Methods: We performed an observational retrospective study with data from 3451 enrolled patients with AP. Serum D-dimer levels were measured upon admission, after $24 \mathrm{~h}$ and during the week after admission by immunoturbidimetry. Univariate and multivariate analyses were used to determine whether elevated D-dimer levels were independently associated with the severity of AP.

Results: Of the 3451 AP patients, 2478 (71.8\%) had serum D-dimer levels measured within $24 \mathrm{~h}$ of hospital admission; 1273 of these patients had D-dimer levels $\leq 2.5 \mathrm{mg} / \mathrm{L}$, and 1205 had D-dimer levels $>2.5 \mathrm{mg} / \mathrm{L}$ (934 patients had mild AP (MAP); 1086, moderately severe AP (MSAP); and 458, severe AP (SAP)). Patients with D-dimer levels $>2.5 \mathrm{mg} / \mathrm{L}(n=1205)$ had higher incidences of SAP ( $75.5 \%$ vs. $24.5 \%)$, acute peripancreatic fluid collection (APFC) (53.3\% vs. 46.7\%), acute necrotic collection (ANC) (72.4\% vs. 27.6\%), pancreatic necrosis (PN) (65.2\% vs. 34.8\%), infected pancreatic necrosis (IPN) (77.7\% vs. 22.8\%), organ failure (OF) (68.5\% vs. 31.5\%), persistent organ failure (POF) $(75.5 \%$ vs. $24.5 \%)$, ICU requirement (70.2\% vs. $29.8 \%)$, and mortality $(79.2 \%$ vs. $20.8 \%)$ than did patients with D-dimer levels $\leq 2.5 \mathrm{mg} / \mathrm{L}(n=1273)$. The multivariate analysis showed that patients with higher serum D-dimer levels had poorer prognoses that worsened over time.

Conclusion: The measurement of D-dimer levels at admission may be useful for risk stratification of AP.

Keywords: Acute pancreatitis, D-dimer, Acute necrotic collection, Multiple organ failure, Prognosis

\section{Background}

Acute pancreatitis (AP) is a common clinical acute abdominal disease that has a narrow therapeutic window. Currently, AP is classified as mild AP (MAP), moderately severe AP (MSAP), and severe AP (SAP) [1]. The majority of patients have a mild form of the disease and recover well, but approximately $20 \%$ develop SAP, which has a high mortality rate (15-35\%) [2], mainly due to pancreatic necrosis, systemic inflammation and persistent multiple organ dysfunction associated with

\footnotetext{
*Correspondence: xialiang79@163.com

† Jianhua Wan and Xiaoyu Yang contributed equally to this work. Department of Gastroenterology, The First Affiliated Hospital of Nanchang University, 17 Yongwaizheng Street, Nanchang, Jiangxi 330006, People's
} Republic of China

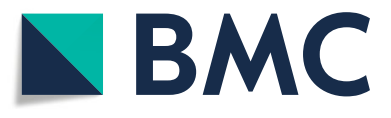

(c) The Author(s). 2019 Open Access This article is distributed under the terms of the Creative Commons Attribution 4.0 International License (http://creativecommons.org/licenses/by/4.0/), which permits unrestricted use, distribution, and reproduction in any medium, provided you give appropriate credit to the original author(s) and the source, provide a link to the Creative Commons license, and indicate if changes were made. The Creative Commons Public Domain Dedication waiver (http://creativecommons.org/publicdomain/zero/1.0/) applies to the data made available in this article, unless otherwise stated. concurrent infections [3-5]. In addition, the annual incidence of AP has increased along with medical costs, and AP is one of the leading causes of in-hospital deaths in developed countries [3, 6-9]. Early treatment, for which the early diagnosis and assessment of AP severity are essential, has been shown to reduce mortality $[10,11]$. Currently, however, no method of effectively detecting disease severity is available [12]. Various laboratory markers [13] have been used to predict AP severity; however, the low accuracy, cumbersome laboratory techniques and high cost associated with these approaches currently limit their clinical application. Furthermore, the existing scoring systems seem to have reached their maximal efficacy [14]. Performing CT upon 
admission solely to assess AP severity is not recommended [15], and radiological tests are expensive to perform. Therefore, new biomarkers are desirable and necessary for better predicting the severity of AP.

Pancreatitis induces the formation of venous thrombosis [16-18]. Thrombosis is a vascular complication of AP and a major cause of AP morbidity and mortality [19]. Notably, in a recent study, Min-Jung Park observed intrapancreatic thrombosis [20]. The early peak in AP mortality is mainly due to the systemic inflammatory response; the circulatory system is in a hypercoagulable state, which may induce the formation of thrombosis and aggravate AP due to tissue ischemia. Abdulla A found that the depletion of platelets decreased cerulein-induced myeloperoxidase (MPO) levels and neutrophil recruitment in the pancreas [21]. Moreover, the administration of heparin alleviated cerulein-mediated pancreatic injury [20]. These studies suggested that thrombosis has a critical impact on the prognosis of AP. D-dimer, a soluble fibrin degradation product, is central to the diagnostic workup of suspected deep-vein thrombosis and pulmonary embolism [22]. Numerous studies have shown that D-dimer serves as a valuable marker of the activation of coagulation and fibrinolysis [23]. Furthermore, D-dimer levels are elevated in a variety of conditions, including atrial fibrillation [24], coronary artery disease [25], and HIV infection [26], suggesting that D-dimer may play an important role in the assessment of AP severity. The use of D-dimer levels as an indicator of disease severity in small samples of patients has recently been reported $[27,28]$, but the quality of the evidence is low. Thus, the association between D-dimer levels and AP severity requires further investigation.

The purposes of this investigation were to define the sensitivity of D-dimer levels for predicting AP severity with our data and to assess the potential of serum D-dimer levels as a marker of disease severity in AP patients.

\section{Methods}

In this observational study, all consecutive patients were retrospectively collected from our single-center hospital between January 1, 2014, and December 31, 2017. The ethics committee of The First Affiliated Hospital of Nanchang University reviewed and approved this study (No. 2011001).

\section{Patient selection}

We collected the data from an electronic medical database in our hospital. Patients who were diagnosed with $\mathrm{AP}$ and had serum D-dimer levels measured within $24 \mathrm{~h}$ of presentation were included in the study. The criteria for a diagnosis of AP include classic abdominal pain, serum amylase and/or lipase and radiographic evidence, which were described in a previous study [10]. The classification of acute pancreatitis is well recognized according to the latest 2012 revision of the Atlanta classification (MAP, SAP and MSAP).

\section{Data collection}

For each patient, age, sex, medical history, admission number, and date were collected as baseline demographic data. Moreover, we collected vital signs of all patients on admission and important laboratory tests, radiological data and clinical outcomes after hospitalization.

\section{Statistical methods}

IBM Statistical Package for Social Sciences (SPSS) software version 20.0 (Chicago, USA) was used to perform the statistical analyses. The results are presented as percentages (\%) or means $\pm \mathrm{SD}$. Comparisons were performed using Student's t test for two groups of independent samples, Cuzick's trend test for multiple groups and the $\chi^{2}$ and Fisher's exact tests for categorical variables. Logistic regression analyses were performed to predict risk factors with categorical dependent variables. Differences were considered to be statistically significant at $P<0.05$.

\section{Results}

Of the 3451 patients with AP, 2478 (71.8\%) had serum D-dimer levels measured within $24 \mathrm{~h}$ of hospital admission. The demographics and clinical characteristics (sex; age; body mass index (BMI); and common etiologies, including biliary dysfunction, hypertriglyceridemia and alcoholism) were similar between the patients with available early serum D-dimer levels $(n=2478)$ and those without $(n=973)$, as shown in Table 1 . No significant differences were observed between the patients with available early serum D-dimer levels and those without in terms of clinical outcomes, including APFC (26.4\% vs. $28.0 \%)$, ANC (17.4\% vs. $15.4 \%)$, PN (22.3\% vs. $24.4 \%)$, POF (18.5\% vs. $17.0 \%)$, and mortality $(2.0 \%$ vs. $1.2 \%$; all $P>0.1$; Table 1).

For the prediction of SAP, the area under the curve (AUC) for serum D-dimer levels was 0.714 $(P<0.001$; Fig. 1). To calculate the accuracy of the serum $D$-dimer levels, an optimal cutoff value of $2.5 \mathrm{mg} / \mathrm{L}$ was used for the prediction of SAP, as shown in Table 2. The 1205 patients with a serum D-dimer level above $2.5 \mathrm{mg} / \mathrm{L}$ demonstrated a poorer prognosis than the 1237 patients with a serum D-dimer level below $2.5 \mathrm{mg} / \mathrm{L}$ (Table 2). Higher incidences of SAP (75.5\%), APFC (53.3\%), ANC (72.4\%), PN (65.2\%), IPN (77.7\%), OF (68.5\%), POF (75.5\%), ICU requirement (70.2\%), and mortality (79.2\%) were observed in the high D-dimer group, and longer median hospital stays (7 vs. 10) and median ICU stays (0 vs. 3) were found in the high D-dimer group. 
Table 1 Comparison of baseline clinical characteristics and outcomes between AP patients with vs. without serum D-dimer drawn in admission to the hospital

\begin{tabular}{llll}
\hline & $\begin{array}{l}\text { D-dimer } \\
\text { measured } \\
\text { Variables }\end{array}$ & $\begin{array}{l}\text { D-dimer not } \\
\text { available } \\
N=2478\end{array}$ & $P$ \\
\hline Male, N (\%) & $1405(56.7)$ & $510(52.4)$ & 0.492 \\
Median age, (IQR) & $51(40-64)$ & $51(40-64)$ & 0.759 \\
Median BMI, (IQR) & $23(21-25)$ & $23(21-25)$ & 0.838 \\
Etiology, N (\%) & & & 0.309 \\
Biliary & $1448(58.4)$ & $587(60.3)$ & \\
Alcoholism & $214(8.6)$ & $70(7.2)$ & \\
Hypertriglyceridemia & $646(26.1)$ & $265(27.2)$ & \\
Others & $170(6.9)$ & $51(5.2)$ & \\
Outcomes & & & 0.314 \\
APFC, N (\%) & $653(26.4)$ & $272(28.0)$ & 0.163 \\
ANC, N (\%) & $431(17.4)$ & $150(15.4)$ & 0.190 \\
PN, N (\%) & $552(22.3)$ & $237(24.4)$ & 0.197 \\
POF, N (\%) & $458(18.5)$ & $165(17.0)$ & 0.119 \\
Mortality, N (\%) & $50(2.0)$ & $12(1.2)$ & \\
\hline
\end{tabular}

$A P$ acute pancreatitis, $N$ number, APFC acute peripancreatic fluid collection, $A N C$ acute necrotic collection, $P N$ Pancreatic necrosis, POF persistent organ failure, IQR Inter Quartile Range
The serum D-dimer level was recorded on different days within 1 week of admission to observe sequential changes in this indicator during the early days following admission to the hospital with AP. The overall D-dimer level gradually increased after admission. In the analysis of AP severity classification, the D-dimer level in the SAP group gradually increased but remained higher than that in the MSAP and MAP groups (all P-trend $<0.001$; Table 3). Similarly, the outcomes of AP, including organ failure and pancreatic necrosis, worsened with higher levels of serum D-dimer (all $P<0.01$; Table 3 ). Thus, the serum D-dimer level seems to be a useful marker for severity classification and outcomes in AP.

A comparison of the baseline clinical characteristics of AP patients by severity classification (MAP vs. MSAP vs. SAP) indicated that the proportion of men in each group was not significantly different $(56.2 \%$ vs. $57.9 \%$ vs. $54.8 \%)$. SAP patients were older and weighed more than either MSAP or MAP patients. An etiology of hypertriglyceridemia was associated with a higher incidence of MSAP (30.2\%) and SAP (29.9\%) (P-trend $<0.001)$ than that of MAP. Glucose (GLU), blood urea nitrogen $(\mathrm{BUN})$, and creatinine levels increased as AP severity increased (P-trend $<0.001 ; \mathrm{P}$-trend $<0.001$; P-trend $<0.001$ ). Furthermore, prothrombin time (PT) and activated partial thromboplastin time (APTT), as markers of

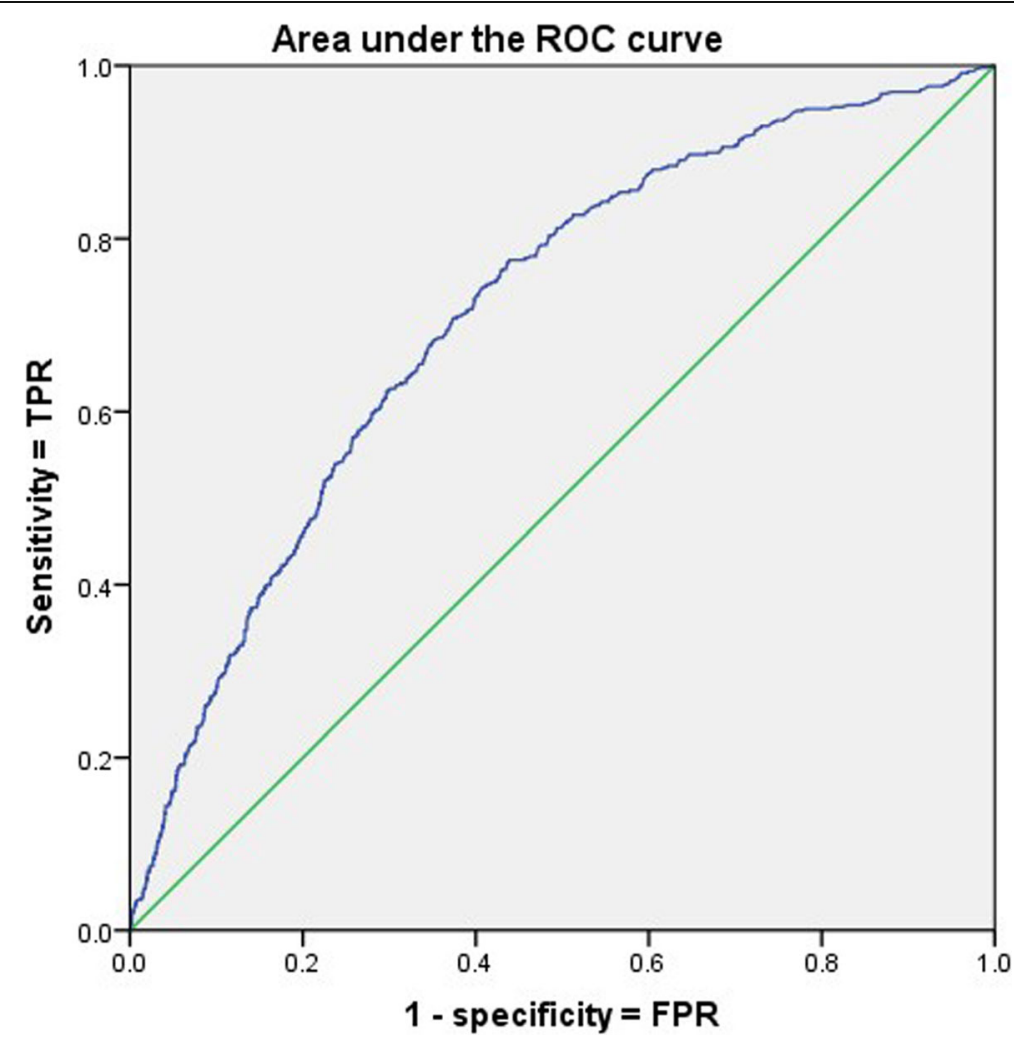

Fig. 1 The area under the curve (AUC) of serum D-dimer levels to predict SAP 
Table 2 Population baseline characteristics between serum Ddimer level categories

\begin{tabular}{llll}
\hline & D-dimer $\leq 2.5$ & D-dimer $>2.5$ & \\
Variable & $N=1273$ & $N=1205$ & $P$ \\
\hline Severity classification, N (\%) & & & $<0.001$ \\
$\quad$ MAP & $667(52.4)$ & $267(22.2)$ & \\
$\quad$ MSAP & $494(38.8)$ & $592(49.1)$ & \\
$\quad$ SAP & $112(8.8)$ & $346(28.7)$ & \\
APFC, N (\%) & $305(24.0)$ & $348(28.9)$ & 0.005 \\
ANC, N (\%) & $119(9.3)$ & $312(25.9)$ & $<0.001$ \\
PN, N (\%) & $192(15.1)$ & $360(29.9)$ & $<0.001$ \\
Infected PN, N (\%) & $31(2.4)$ & $108(9.0)$ & $<0.001$ \\
OF, N (\%) & $258(20.3)$ & $561(46.6)$ & $<0.001$ \\
POF, N (\%) & $112(8.8)$ & $346(28.7)$ & $<0.001$ \\
Median hospital days, (IQR) & $7(5-10)$ & $10(6-16)$ & $<0.001$ \\
Median ICU days, (IQR) & $0(0-2)$ & $3(0-7)$ & $<0.001$ \\
Admission to ICU, N (\%) & $203(15.9)$ & $479(39.8)$ & $<0.001$ \\
Mortality, N (\%) & $10(0.8)$ & $38(3.2)$ & $<0.001$ \\
\hline
\end{tabular}

$N$ number, MAP mild acute pancreatitis, MSAP moderately severe acute pancreatitis, SAP severe acute pancreatitis, APFC acute peripancreatic fluid collection, $A N C$ acute necrotic collection, $P N$ Pancreatic necrosis, OF organ failure, $P O F$ persistent organ failure, IQR Inter Quartile Range

blood coagulation, increased as AP severity increased (P-trend $<0.001 ;$ P-trend $<0.001$ ). The number of cases (100; $21.8 \%)$ of infected pancreatic necrosis observed in the SAP group was higher than that observed in the MSAP group (3.5\%) (P-trend $<0.001)$. Moreover, a higher incidence of mortality was observed in the SAP group

Table 3 Table showing D-dimer levels in various groups on different days of admission to the hospital within 1 week

\begin{tabular}{clllll}
\hline \multicolumn{7}{c}{ Day 1} & Day 2 & Day 3 & Day 5 & Day 7 \\
Variable & $N=2478$ & $N=1413$ & $N=604$ & $N=558$ & $N=385$ \\
\hline Severity classification & & & & \\
MAP & $2.69 \pm 5.58$ & $2.70 \pm 4.41$ & $3.69 \pm 4.65$ & $4.03 \pm 6.34$ & $5.66 \pm 6.42$ \\
MSAP & $4.46 \pm 5.29$ & $5.09 \pm 6.23$ & $5.59 \pm 4.96$ & $6.69 \pm 5.48$ & $7.00 \pm 5.41$ \\
SAP & $7.21 \pm 8.90$ & $7.21 \pm 7.09$ & $8.03 \pm 6.66$ & $9.55 \pm 8.93$ & $11.10 \pm 11.49$ \\
$P_{\text {trend }}$ & $<0.001$ & $<0.001$ & $<0.001$ & $<0.001$ & $<0.001$ \\
Organ failure & & & & \\
NO & $3.30 \pm 5.20$ & $3.53 \pm 4.65$ & $4.47 \pm 4.64$ & $5.61 \pm 5.61$ & $6.17 \pm 4.77$ \\
YES & $6.33 \pm 7.98$ & $6.76 \pm 7.56$ & $7.42 \pm 6.32$ & $8.70 \pm 8.29$ & $10.36 \pm 10.64$ \\
$P$ & $<0.001$ & $<0.001$ & $<0.001$ & $<0.001$ & $<0.001$ \\
Pancreatic necrosis & & & & \\
NO & $3.96 \pm 6.56$ & $4.36 \pm 5.99$ & $5.57 \pm 6.08$ & $6.52 \pm 6.69$ & $7.26 \pm 7.08$ \\
YES & $5.51 \pm 6.11$ & $5.99 \pm 6.46$ & $6.91 \pm 5.04$ & $9.26 \pm 8.52$ & $10.93 \pm 11.07$ \\
$P$ & $<0.001$ & $<0.001$ & 0.007 & $<0.001$ & $<0.001$ \\
\hline
\end{tabular}

$N$ number, MAP mild acute pancreatitis, MSAP moderately severe acute pancreatitis, SAP severe acute pancreatitis than in the MSAP and MAP groups $(9.8 \%$ vs. $0.3 \%$ vs. $0 \%$; P-trend $<0.001$ ), as shown in Table 4.

The logistic multivariate regression analysis showed an association between serum D-dimer level and complications after adjusting for age, sex, pancreatitis etiology, smoking status, and alcohol use status. As shown in Table 5, a higher serum D-dimer level was independently associated with pancreatitis prognosis and complications, including APFC, ANC, pancreatic necrosis, infected pancreatic necrosis, organ failure, persistent organ failure, ICU admission, and mortality.

\section{Discussion}

This study is a single-center observational retrospective analysis that evaluated simple laboratory parameters as predictors of SAP. Here, we studied the diagnostic value of D-dimer levels for predicting AP severity. Many indicators are currently available for predicting SAP and include $\mathrm{C}$-reactive protein (CRP) and BUN, the most widely used parameters for the assessment of AP severity; however, none of them differ significantly within 24 $\mathrm{h}$ after the onset of symptoms. Levels of serum lipase and amylase, two major markers for pancreatitis, have also been shown to be disproportionate to the severity of the disease [8]. Thus, these indicators seem to have reached their maximal efficacy [2, 8, 13, 14, 29, 30]. New indicators for assessing the severity of AP have been reported in recent studies [28, 31-33]. However, these detection indicators are expensive and difficult to operate. In particular, the specific mechanism remains uncertain, and additional studies are needed. Interleukin-6 significantly improves the predictive value for severe acute pancreatitis but is difficult to detect [34]. With the advent of a fully automated assay, IL- 6 is currently being used clinically in some hospitals [35]. Therefore, continuing to study valuable markers is necessary. The D-dimer level, which is a marker of the activation of coagulation and fibrinolysis, provides a rapid assessment of thrombotic activity and safely excludes patients with suspected venous thromboembolism (VTE) based on the clinical decision rule $[36,37]$. In addition, D-dimer has been widely used in clinical settings because it is convenient and stable.

The possibility that D-dimer levels can predict the severity of AP may be explained by the following pathogenic mechanism. At the onset of AP, the abnormal activation of pancreatic enzymes results in inflammation and injury to the pancreas, which then induces thrombosis and further aggravates the injury [38]. Two mortality peaks occur in patients with AP, namely, early mortality due to the effects of systemic inflammatory response syndrome (SIRS) and multiple organ dysfunction syndrome (MODS) and late mortality caused by the effects of MODS combined with sepsis following pancreatic 
Table 4 Comparison of baseline clinical characteristics and outcomes between AP patients on severity classification

\begin{tabular}{|c|c|c|c|c|}
\hline & MAP & MSAP & SAP & \\
\hline Variable & $N=934$ & $N=1086$ & $N=458$ & $P_{\text {trend }}$ \\
\hline Male, $N(\%)$ & $525(56.2)$ & $629(57.9)$ & $251(54.8)$ & 0.492 \\
\hline Median age, (IQR) & $51(41-64)$ & $49(39-62)$ & $55(44-66)$ & $<0.001$ \\
\hline $\mathrm{BMl}$ & $22.74 \pm 3.38$ & $23.55 \pm 3.54$ & $23.77 \pm 3.56$ & $<0.001$ \\
\hline \multicolumn{5}{|l|}{ Etiology, N (\%) } \\
\hline Biliary & $569(60.9)$ & $603(55.5)$ & $276(60.3)$ & 0.034 \\
\hline Alcoholism & $67(7.2)$ & $104(9.6)$ & $43(9.4)$ & 0.130 \\
\hline Hypertriglyceridemia & $181(19.4)$ & $328(30.2)$ & $137(29.9)$ & $<0.001$ \\
\hline Smoker, $N(\%)$ & $175(18.7)$ & $230(21.2)$ & $104(22.7)$ & 0.178 \\
\hline Alcoholism, N (\%) & 155 (16.6) & $224(20.6)$ & $112(24.5)$ & 0.002 \\
\hline $\mathrm{GLU}, \mathrm{mmol} / \mathrm{L}$ & $7.17 \pm 3.05$ & $8.59 \pm 4.13$ & $9.81 \pm 5.22$ & $<0.001$ \\
\hline $\mathrm{BUN}, \mathrm{mmol} / \mathrm{L}$ & $5.02 \pm 2.88$ & $5.61 \pm 3.09$ & $9.71 \pm 7.37$ & $<0.001$ \\
\hline Creatinine, $\mathrm{mmol} / \mathrm{L}$ & $67.37 \pm 51.77$ & $69.07 \pm 42.42$ & $130.85 \pm 128.10$ & $<0.001$ \\
\hline PT, s & $12.21 \pm 7.60$ & $12.62 \pm 6.69$ & $13.97 \pm 7.60$ & $<0.001$ \\
\hline APTT, s & $29.61 \pm 10.58$ & $31.41 \pm 14.81$ & $36.13 \pm 14.40$ & $<0.001$ \\
\hline D-dimer, mg/L & $2.69 \pm 5.88$ & $4.46 \pm 5.29$ & $7.21 \pm 8.90$ & $<0.001$ \\
\hline Median APACHEII, (IQR) & $5(3-8)$ & $6(4-9)$ & $10(8-13)$ & $<0.001$ \\
\hline $\mathrm{PN}, N(\%)$ & 0 & $328(30.2)$ & $220(48.0)$ & $<0.001$ \\
\hline Infected PN, N (\%) & 0 & $38(3.5)$ & $100(21.8)$ & $<0.001$ \\
\hline Median hospital days (IQR) & $6(4-8)$ & $9(6-14)$ & $14(9-25)$ & $<0.001$ \\
\hline Median ICU days, (IQR) & $0(0)$ & $0(0-4)$ & $7(4-15)$ & $<0.001$ \\
\hline Admission to ICU, N (\%) & $74(7.9)$ & $259(23.8)$ & $349(76.2)$ & $<0.001$ \\
\hline Mortality, N (\%) & $0(0)$ & $3(0.3)$ & $45(9.8)$ & $<0.001$ \\
\hline
\end{tabular}

MAP mild acute pancreatitis, MSAP moderately severe acute pancreatitis, SAP severe acute pancreatitis, $N$ number, GLU blood glucose, BUN Blood urea nitrogen, PT prothrombin time, APTT activated partial thromboplastin time, PN Pancreatic necrosis, IQR Inter Quartile Range

necrosis and infection. Systemic inflammation is a common risk factor for the development of VTE [22]. Clearly, crosstalk occurs between the inflammatory response and the clotting reaction. A disturbance of the coagulation system has long been thought to be implicated in the

Table 5 Multivariate analysis showing association of serum D-dimer level with complications after adjusting for age, sex, pancreatitis etiology, smoker, alcoholism

\begin{tabular}{llll}
\hline Complication & B & OR & $P$ \\
\hline APFC & 0.27 & 1.30 & 0.004 \\
ANC & 1.25 & 3.49 & $<0.001$ \\
PN & 0.90 & 2.46 & $<0.001$ \\
Infected PN & 1.37 & 3.94 & $<0.001$ \\
OF & 1.21 & 3.35 & $<0.001$ \\
POF & 1.32 & 3.74 & $<0.001$ \\
Admission to ICU & 1.23 & 3.41 & $<0.001$ \\
Mortality & 1.34 & 4.04 & $<0.001$ \\
\hline
\end{tabular}

$B$ regression coefficient, $A P F C$ acute peripancreatic fluid collection, $A N C$ acute necrotic collection, $P N$ Pancreatic necrosis, $O F$ organ failure, $P O F$ persistent organ failure pathogenesis of systemic and local pancreatitis complications [18]. In addition to the thrombosis of the pancreas itself, pancreatitis leading to coronary venous thrombosis and splanchnic vein thrombosis have also been reported $[39,40]$. D-dimer, a common indicator of thrombosis, may play an important role in the assessment of AP severity by monitoring pancreatic and extrapancreatic thrombosis. In our study, AP patients with high D-dimer levels had a higher incidence of pancreatic necrosis and organ failure than did patients with normal D-dimer levels. This result suggested that the severity of AP is closely associated with the presence of inflammation and thrombosis.

Few studies have been conducted to assess the value of D-dimer, a fibrin degradation product, in predicting AP severity. In those retrospective studies, patients with SAP had higher D-dimer levels than did those with MAP, suggesting that D-dimer levels had moderate diagnostic accuracy in predicting MSAP and exhibited excellent diagnostic accuracy in predicting SAP [27, 28, 41, 42]. In a single-center, retrospective study including 71 patients, Cécile Gomercic [41] obtained results implying that either alone or in combination with the CRP level, 
the $\mathrm{D}$-dimer level may be a useful early predictive biomarker of AP. Aleksandra Boskovic [42] reported that the D-dimer level may be a simple clinical predictor of pediatric AP severity and local complications in a small cohort of 36 patients with AP.

Similar to these previous results, our results implied that the D-dimer level may be a potential biomarker for predicting AP severity. Compared with prior reports, our study has several strengths, including the continuous nature of patient enrollment and the large sample size. In addition, we recorded a sequential change in the serum D-dimer level in the early days (within 1 week) after admission to the hospital. The logistic multivariate regression analysis showed that the D-dimer level upon admission was independently associated with early prediction of AP outcome. In addition to the D-dimer level, the blood coagulation markers PT and APTT were found to increase with greater AP severity (P-trend $<0.001 ; \mathrm{P}$-trend $<0.001$ ).

This study has several potential limitations. First, D-dimer could not be detected in some of the samples obtained upon admission; therefore, the measurement of D-dimer levels may be subject to selection bias. To counter this potential limitation, a head-to-head comparison of patients having available $\mathrm{D}$-dimer values with those not having available D-dimer values was performed. No difference in clinical outcomes, including persistent organ failure, was found between the two groups. Second,

the D-dimer level is affected by many factors; any process (including pregnancy, inflammation, cancer, and surgery) that increases fibrin production or breakdown also increases D-dimer levels. In addition, D-dimer levels and age are closely related [43]. Furthermore, the magnitude of the unit of measure for the D-dimer level varies widely across laboratories using the same assays, and an accepted standardization for D-dimer concentration across assay types is lacking. The optimal D-dimer test methodology and associated threshold value for detecting AP remain uncertain. Consequently, the results available for one assay cannot simply be extrapolated to other assays, even those using similar formats [43, 44].

\section{Conclusions}

In addition to excluding VTE, D-dimer testing may play a critical role in the prediction of AP severity. However, the wide variation in the types and operating characteristics of D-dimer assays means that the results of studies that used one assay cannot be extrapolated to studies that used another assay, which limits the use of multicenter studies. Much work remains to be done to standardize the performance and reporting of D-dimer assays as well as to translate the results of D-dimer studies into clinical practice.

\section{Abbreviations}

ANC: Acute necrotic collection; AP: Acute pancreatitis; APFC: Acute peripancreatic fluid collection; APTT: Activated partial thromboplastin time; AUC: Area under the curve; BUN: Blood urea nitrogen; CRP: C-reactive protein; GLU: Glucose; IPN: Infected pancreatic necrosis; MAP: Mild AP; MODS: Multiple organ dysfunction syndrome; MPO: Myeloperoxidase; MSAP: Moderately severe AP; OF: Organ failure; PN: Pancreatic necrosis; POF: Persistent organ failure; PT: Prothrombin time; SAP: Severe AP; SIRS: Systemic inflammatory response syndrome; VTE: Venous thromboembolism

\section{Acknowledgments}

Not applicable.

\section{Funding}

The study design, data collection, analysis and interpretation of data, writing manuscript were funded by the National Natural Science Foundation of China (No: 81460130 and 81760121); the Graduate Teaching Library Construction Project of Nanchang University (No. 9202-0210210802); and the Graduate Innovation fund of Jiangxi Province (No. YC2017-B016).

\section{Availability of data and materials}

All data generated or analyzed during this study are included in this published article [and its supplementary information files].

\section{Authors' contributions}

$J H W$ and $X Y Y$ conceived the study; JH W, WH H, Y Z1, L X and NH L participated in the design; $Y Z 1, Y Z 2, H Z$ and $P L$ collected the data; and $J H$ $W$ and $W H H$ performed statistical analyses. JH W and $X Y Y$ drafted the manuscript. $L X$ and $\mathrm{NH} L$ edited and checked the manuscript. All of the authors have read and approved the final manuscript.

\section{Ethics approval and consent to participate}

The study was approved by the ethics committee of The First Affiliated Hospital of Nanchang University (No. 2011001). Informed consent by verbal was obtained from all participants approved by the ethics committee.

\section{Consent for publication}

Not applicable.

Competing interests

The authors declare that they have no competing interests.

\section{Publisher's Note}

Springer Nature remains neutral with regard to jurisdictional claims in published maps and institutional affiliations.

Received: 3 February 2019 Accepted: 18 April 2019

Published online: 02 May 2019

\section{References}

1. Banks PA, Bollen TL, Dervenis C, Gooszen HG, Johnson CD, Sarr MG, et al. Classification of acute pancreatitis--2012: revision of the Atlanta classification and definitions by international consensus. Gut. 2013;62(1):102-11.

2. van Dijk SM, Hallensleben NDL, van Santvoort HC, Fockens P, van Goor H, Bruno MJ, et al. Acute pancreatitis: recent advances through randomised trials. Gut. 2017;66(11):2024-32.

3. Peery AF, Dellon ES, Lund J, Crockett SD, McGowan CE, Bulsiewicz WJ, et al. Burden of gastrointestinal disease in the United States: 2012 update. Gastroenterology. 2012;143(5):1179-87 e1-3.

4. Pandol SJ, Saluja AK, Imrie CW, Banks PA. Acute pancreatitis: bench to the bedside. Gastroenterology. 2007;132(3):1127-51.

5. Whitcomb DC. Genetic risk factors for pancreatic disorders. Gastroenterology. 2013;144(6):1292-302.

6. Frossard J-L, Steer ML, Pastor CM. Acute pancreatitis. Lancet. 2008;371(9607): 143-52.

7. Yadav D, Lowenfels $A B$. The epidemiology of pancreatitis and pancreatic cancer. Gastroenterology. 2013;144(6):1252-61.

8. Lankisch PG, Apte M, Banks PA. Acute pancreatitis. Lancet. 2015:386(9988): $85-96$. 
9. Roberts SE, Morrison-Rees S, John A, Williams JG, Brown TH, Samuel DG. The incidence and aetiology of acute pancreatitis across Europe. Pancreatology. 2017;17(2):155-65

10. Tenner S, Baillie J, DeWitt J, Vege SS. American College of G. American College of Gastroenterology guideline: management of acute pancreatitis. Am J Gastroenterol. 2013;108(9):1400-15.

11. Fisher JM, Gardner TB. The "golden hours" of management in acute pancreatitis. Am J Gastroenterol. 2012;107(8):1146-50.

12. Gomatos IP, Xiaodong X, Ghaneh P, Halloran C, Raraty M, Lane B, et al. Prognostic markers in acute pancreatitis. Expert Rev Mol Diagn. 2014;14(3):333-46.

13. Staubli SM, Oertli D, Nebiker CA. Laboratory markers predicting severity of acute pancreatitis. Crit Rev Clin Lab Sci. 2015;52(6):273-83.

14. Mounzer R, Langmead CJ, Wu BU, Evans AC, Bishehsari F, Muddana V, et al. Comparison of existing clinical scoring systems to predict persistent organ failure in patients with acute pancreatitis. Gastroenterology. 2012;142(7):1476-82

15. Bollen TL, Singh VK, Maurer R, Repas K, van Es HW, Banks PA, et al. A comparative evaluation of radiologic and clinical scoring systems in the early prediction of severity in acute pancreatitis. Am J Gastroenterol. 2012;107(4):612-9.

16. Berzigotti A, Garcia-Criado A, Darnell A, Garcia-Pagan JC. Imaging in clinica decision-making for portal vein thrombosis. Nat Rev Gastroenterol Hepatol. 2014;11(5):308-16.

17. Harris S, Nadkarni NA, Naina HV, Vege SS. Splanchnic vein thrombosis in acute pancreatitis: a single-center experience. Pancreas. 2013;42(8):1251-4.

18. Maeda K, Hirota M, Ichihara A, Ohmuraya M, Hashimoto D, Sugita H, et al. Applicability of disseminated intravascular coagulation parameters in the assessment of the severity of acute pancreatitis. Pancreas. 2006;32(1):87-92.

19. Lisman T, Porte RJ. Activation and regulation of hemostasis in acute live failure and acute pancreatitis. Semin Thromb Hemost. 2010;36(4):437-43.

20. Park MJ, Iyer S, Xue X, Bragazzi Cunha J, Gu S, Moons D, et al. HIF1-alpha regulates acinar cell function and response to injury in mouse pancreas. Gastroenterology. 2018;154(6):1630-4 e3.

21. Abdulla A, Awla D, Hartman H, Rahman M, Jeppsson B, Regner S, et al. Role of platelets in experimental acute pancreatitis. Br J Surg. 2011;98(1):93-103.

22. Di Nisio M, van Es N, Büller HR. Deep vein thrombosis and pulmonary embolism. Lancet. 2016:388(10063):3060-73.

23. Weitz JI, Fredenburgh JC, Eikelboom JW. A test in context: D-dimer. J Am Coll Cardiol. 2017:70(19):2411-20.

24. Hijazi Z, Oldgren J, Siegbahn A, Wallentin L. Application of biomarkers for risk stratification in patients with atrial fibrillation. Clin Chem. 2017;63(1):152-64.

25. Watanabe H, Horita N, Shibata Y, Minegishi S, Ota E, Kaneko T. Diagnostic test accuracy of D-dimer for acute aortic syndrome: systematic review and meta-analysis of 22 studies with 5000 subjects. Sci Rep. 2016;6:26893.

26. Triant VA, Lee H, Hadigan C, Grinspoon SK. Increased acute myocardial infarction rates and cardiovascular risk factors among patients with human immunodeficiency virus disease. J Clin Endocrinol Metab. 2007;92(7):2506-12.

27. Ke L, Ni HB, Tong ZH, Li WQ, Li N, Li JS. D-dimer as a marker of severity in patients with severe acute pancreatitis. J Hepatobiliary Pancreat Sci. 2012;19(3):259-65.

28. Yang N, Hao J, Zhang D. Antithrombin III and D-dimer levels as indicators of disease severity in patients with hyperlipidaemic or biliary acute pancreatitis. J Int Med Res. 2017:45(1):147-58.

29. Ismail OZ, Bhayana V. Lipase or amylase for the diagnosis of acute pancreatitis? Clin Biochem. 2017;50(18):1275-80.

30. Di MY, Liu H, Yang ZY, Bonis PA, Tang JL, Lau J. Prediction models of mortality in acute pancreatitis in adults: a systematic review. Ann Intern Med. 2016;165(7):482-90

31. Expression of concern--Probiotic prophylaxis in predicted severe acute pancreatitis: a randomised, double-blind, placebo-controlled trial. Lancet. 2010;375(9718):875-876.

32. Tan C, Ling Z, Huang Y, Cao Y, Liu Q, Cai T, et al. Dysbiosis of intestinal microbiota associated with inflammation involved in the progression of acute pancreatitis. Pancreas. 2015;44(6):868-75.

33. Dumnicka P, Sporek M, Mazur-Laskowska M, Ceranowicz P, Kuzniewski M, Drozdz R, et al. Serum soluble Fms-like tyrosine kinase 1 (sFlt-1) predicts the severity of acute pancreatitis. Int J Mol Sci. 2016;17(12):2038.

34. Jain S, Midha S, Mahapatra SJ, Gupta S, Sharma MK, Nayak B, et al. Interleukin-6 significantly improves predictive value of systemic inflammatory response syndrome for predicting severe acute pancreatitis. Pancreatology. 2018;18(5):500-6.

35. Kolber W, Dumnicka P, Maraj M, Kusnierz-Cabala B, Ceranowicz P, Pedziwiatr $M$, et al. Does the automatic measurement of interleukin 6 allow for prediction of complications during the first $48 \mathrm{~h}$ of acute pancreatitis? Int J Mol Sci. 2018;19(6):1820.

36. Lucassen W, Geersing GJ, Erkens PM, Reitsma JB, Moons KG, Buller H, et al. Clinical decision rules for excluding pulmonary embolism: a meta-analysis. Ann Intern Med. 2011;155(7):448-60.

37. Geersing GJ, Zuithoff NP, Kearon C, Anderson DR, Ten Cate-Hoek AJ, Elf JL, et al. Exclusion of deep vein thrombosis using the Wells rule in clinically important subgroups: individual patient data meta-analysis. BMJ. 2014;348:91340.

38. Kakafika A, Papadopoulos V, Mimidis K, Mikhailidis DP. Coagulation, platelets, and acute pancreatitis. Pancreas. 2007:34(1):15-20.

39. Gonzelez HJ, Sahay SJ, Samadi B, Davidson BR, Rahman SH. Splanchnic vein thrombosis in severe acute pancreatitis: a 2-year, single-institution experience. HPB (Oxford). 2011;13(12):860-4.

40. Sanghvi $S$, Waqar F, Effat M. Coronary thrombosis in acute pancreatitis. J Thromb Thrombolysis. 2019;47(1):157-61.

41. Gomercic C, Gelsi E, Van Gysel D, Frin AC, Ouvrier D, Tonohouan M, et al. Assessment of D-dimers for the early prediction of complications in acute pancreatitis. Pancreas. 2016:45(7):980-5.

42. Boskovic A, Pasic S, Soldatovic I, Milinic N, Stankovic I. The role of D-dimer in prediction of the course and outcome in pediatric acute pancreatitis. Pancreatology. 2014;14(5):330-4.

43. Linkins LA, Takach Lapner S. Review of D-dimer testing: good, bad, and ugly. Int J Lab Hematol. 2017;39(Suppl 1):98-103.

44. Riley RS, Gilbert AR, Dalton JB, Pai S, McPherson RA. Widely used types and clinical applications of D-dimer assay. Lab Med. 2016;47(2):90-102.
Ready to submit your research? Choose BMC and benefit from:

- fast, convenient online submission

- thorough peer review by experienced researchers in your field

- rapid publication on acceptance

- support for research data, including large and complex data types

- gold Open Access which fosters wider collaboration and increased citations

- maximum visibility for your research: over $100 \mathrm{M}$ website views per year

At $\mathrm{BMC}$, research is always in progress.

Learn more biomedcentral.com/submissions 